\title{
La fusión dialéctica de Las direcciones del método
}

\author{
The dialectic fusion of the directions of method \\ FERNANDO HAYA \\ IES Hermanos Machado (España)
}

Recibido: 22-10-2013 Aceptado definitivamente: 04-04-2014

\section{RESUMEN}

Examina el empleo dialéctico de las que se consideran principales modalidades del pensamiento en relación con el método: la articulación del tiempo, la reflexión negativa y el pensar como ejercicio en dependencia de la libertad trascendental. Concluye que Hegel establece una fusión entre tales direcciones principales del método con vistas al establecimiento de su comienzo absoluto. La fusión se opera como reducción negativa de la forma inteligible del tiempo, que queda expresada como repulsión del uno negativo.

\section{PALABRAS CLAVES}

MÉTODO, TIEMPO, HEGEL, POLO, KANT, NEGACIÓN, UNO REPELENTE.

\begin{abstract}
It examines the dialectical use of the considered major modes of thought in relation to the method: the articulation of time, the negative reflection, and thinking in dependence of transcendental freedom. Hegel provides a fusion between these main directions of the method with a view to establishing their absolute beginning. The merger is operated as a negative reduction of the intelligible form of time, which is expressed as a negative repulsion.

KEY WORDS

METHOD, TIME, HEGEL, POLO, KANT, NEGATION, ONE REPELLENT.
\end{abstract}




\section{LA REDUCCIÓN METÓDICA DEL TIEMPO}

Mi PROPÓSITO EN ESTAS PÁGINAS ES mostrar el nervio que anima la dialéctica, considerada por Hegel método de la filosofía primera. Creo seguir a Polo ${ }^{1}$ con la distinción entre direcciones metódicas de principio. Entiendo por tales, modalidades ejecutivas -metódicas- del pensamiento. El ejercicio en tensión distintiva de las direcciones metódicas de principio equivale al abandono del límite mental.

Las direcciones metódicas de principio aquí consideradas son éstas: la articulación del tiempo, la reflexión negativa o generalizante, y la modalidad ejecutiva del pensar a cuyo cargo corre la exposición libre del método.

Llamo a la libertad trascendental instancia exponente del método en atención a su equivalencia con el ser de la persona, el núcleo del saber, del que dependen los hábitos y operaciones intelectuales. La exposición libre del método puede describirse como un cierto recorrido descendente que acompaña o asiste desde la libertad trascendental. El descenso metódico en cuestión manifiesta la dependencia del saber respecto de su núcleo personal. La instancia exponente del método es también su dimensión hegemónica, es decir, la dirección de principio más excelente. No de tal modo, sin embargo, que su recorrido prescinda del otro par de direcciones. Por el contrario, Hegel propone la fusión, el solapamiento en una sola, de esta triple dirección del método.

Visto desde la primera, el método es regreso, repliegue en ajuste de la insuficiencia del comienzo intelectual. El comienzo intelectual es la abstracción. La dirección metódica incoativa no es la propia operación abstractiva sino su iluminación habitual. Manifestar la insuficiencia del comienzo equivale a advertir el límite en condiciones que permitan su abandono. La advertencia en cuestión se ejerce como pensar metódicamente distintivo de direcciones metódicas de principio.

El comienzo intelectual es la coincidencia en el abstracto, la mismidad de conocer y conocido. La mismidad es introducida por la presencia mental, descrita como la articulación del tiempo entero. El comienzo metódico es la manifestación declarativa de la insuficiencia en el comienzo abstracto. Desde su dirección incoativa, el método ajusta la insuficiencia que concierne a la articulación abstracta del tiempo. Hay tal ajuste si se explicita la conexión de la forma del tiempo entero con las articulaciones parciales de la temporalidad, obtenidas en los niveles intencionales inferiores. Insisto en que este ajuste metódico exige poner en juego el otro par de direcciones metódicas de principio. En cambio, la dialéctica endosa todo el ejercicio metódico al pensar negativo, que barre y arrastra consigo a las otras direcciones del método.

1 Cfr., Polo, L., El acceso al ser, EUNSA, Pamplona, 2004 (2 ed.). 
A la iluminación de la insuficiencia del comienzo la denomino reducción metódica del tiempo. Reducción metódica, porque desaloja la temporalidad del método y la proyecta hacia la exclusiva posición de tema, ni supuesto ni confuso. La reducción metódica es el desocultamiento de la suposición del tiempo; remoción del lugar en que el dominio del tiempo se oculta y traba el método. La reducción metódica del tiempo es desasimiento de la forma temporal inteligible plena. El adelantamiento con que la presencia se impone es límite, tenaza del pensar. La advertencia del límite se vierte como la noticia de que la consistencia del tiempo entero -su suposición- detiene el avance hacia los primeros principios.

La reducción metódica alcanza el genuino estatuto del tiempo, y por lo tanto el del comienzo en sentido temporal. Permite precisar el valor gnoseológico que haya de asignarse al principio como comienzo. Eso significa deshacer la confusión fácilmente deslizada, tanto desde el esquema imaginativo temporal (la sucesión), como de parte del estatuto inteligible del objeto pensado (la mismidad).

La doble confusión está de suyo entrelazada según una razón positiva. Porque en efecto la forma inteligible del tiempo -la presencia- expande la forma de la sucesión temporal, por decirlo así. La expansión del tiempo sucesivo equivale a su unidad inteligible. De acuerdo con esta unidad de la articulación temporal plena se obtiene objeto intelectual. La operación incoativa, la abstracción, versa sobre el fantasma imaginativo, que es su condición antecedente. El modo de semejante versar es la exposición del contenido real -imaginativamente representado- a la luz de la presencia mental. Eso equivale al coextenderse de la presencia con la forma sucesiva del tiempo, en el sentido de recorrerla o abarcarla.

El comienzo temporal se toma como principio en el orden del tiempo; quiere decirse: del tiempo dado en forma de la sucesión, desde el antes al después. El rango de principio en cuestión - el asignado al comienzo- se amortigua a raíz de su inclusión bajo la forma del tiempo entero. Metódicamente eso quiere decir: más allá del valor que la presencia introduce, cede la exigencia metafísica del tiempo.

En definitiva: en su desplomarse, el estatuto gnoseológico del tiempo arrastra consigo al comienzo, al principio de la secuencia temporal. En este sentido se afirma aquí que la reducción del tiempo es el comienzo metódico de la filosofía. La filosofia comienza por echar cuentas con la insuficiencia del comienzo intelectual. Ahora bien: si el comienzo intelectual no se traza en la secuencia temporal, mucho menos el comienzo metódico.

El pensar mismo admite la descripción de comienzo siempre que se precise lo siguiente: el pensar como comienzo connota un plus, un añadido precisamente referido al tiempo. Pensar es comenzar justamente en salto. El salto en cuestión es relativo al comienzo que -en cambio- se emplaza y fija, como por fuerza el inicio con relación a la secuencia. Eso, entre otras cosas, significa que la presencia mental articula el tiempo entero. Si el pensar se aferrara como comienzo de 
secuencia, no abrazaría, no articularía en plenitud. En cambio, esta como expansión de la presencia exhibe la diferencia, de tal modo que el mostrar se refiere a la vigencia que traspasa el tiempo. Alzar la intelección es salto sobre el tiempo; algo esencialmente distinto del simple engarce de piezas o trozos dentro de un esquema previo tácito e inamovible. Entender es traspasar el tiempo de modo que su forma unitiva no se interponga ni estorbe; de manera que esta forma del tiempo caiga y se funda entera con la transparencia que exhibe.

La insistencia en este punto es capital, porque sin aquilatamiento del valor específico de la intelección -y ciertamente, con referencia al tiempo-, la filosofía no alcanza su cota. La altura de esa cota se tiende en efecto como repliegue sobre el primer comienzo. No se introduce por lo tanto aquí-sino más bien $a$ partir de aquí- la línea de discrepancia con relación a Hegel. Tanto en Hegel como en Polo el método puede decirse reducción metódica del tiempo. Tanto en un pensador como en otro el tiempo exige reducción a su forma entera con vistas a la disposición del método, que es su avance. La diferencia entre los respectivos planteamientos es, a mi modo de ver, la siguiente:

En Polo, la reducción metódica deprime el estatuto metafísico del tiempo sin eliminar su contenido, lo que equivale a evitar la supresión del tiempo mismo. En Hegel, por el contrario, la reducción metódica del tiempo plantea a mi juicio una imposibilidad: no solo confinar el tiempo a su forma -hasta aquí habría acuerdo-, sino desarticular esta misma forma con vistas a la reposición que determina su propio contenido.

El tiempo según Polo es real puesto que establece la vigencia correlativa del movimiento más allá -digamos- del introducirse la presencia mental. El abandono del límite pugna $^{2}$ con la articulación del tiempo entero. Esto quiere decir: el método retrocede desde la antecedencia en orden a discernir la vigencia real del tiempo. En Polo, pues, el confinamiento temático del tiempo es correlativo con el retroceso metódico hasta el primer comienzo (de la vida intelectual), pero sin supresión del contenido real temporal. La manifestación de la forma del tiempo entero no elimina ni suplanta su contenido; más bien lo abarca en cuanto desoculta su articulación inteligible. ${ }^{3}$

Abrazar la plenitud formal del tiempo sin eliminar el contenido equivale a reparar en que la intelección no funda -no pone a sus expensas- lo entendido-. El entender exime. Eximir confiere un nuevo estatuto a lo eximido según el cual un nuevo comienzo queda también instaurado. Entender es traer a un nuevo

2 Cfr., Polo, L., Curso de teoría del conocimiento, IV, EUNSA, Pamplona, 2004 ( $\left.2^{\mathrm{a}} \mathrm{ed}.\right)$, pp. 15-40.

3 Para describir la articulación del tiempo entero empleo las expresiones plenitud formal del tiempo y entera consistencia de lo real. Distingo entre esta consistencia -que es objetivay el valor metafísico -de principio- que pertenece al ser temporal. Esta última adjetivación, en sentido metafísico, no alude a la entera consistencia de lo real sino al primer principio descrito por Polo en términos de comienzo que no cesa ni es seguido. 
comienzo: en este sentido -con relación al tiempo- entender es desaferrar la fijación en el comienzo ya dado según el discurrir sucesivo. Con relación a la forma entendida, el nuevo comienzo despeja su indefinición, el desparramarse valga la expresión- de la determinación real temporal.

Basta con la advertencia de este eximir como inicio del método. El camino esbozado más allá se traza contramano, en suplantación. Eximir del comienzo dado no es sustituirlo, no es posición de tal comienzo. Desde la perspectiva del método, sobra con manifestar la plenitud formal del tiempo. La presencia mental preserva el contenido de realidad toda vez que no se añade a él -en este sentido, sobra- sino que se conmesura. La exención del comienzo dado es el traslucir de su contenido. ${ }^{4}$

La diferencia real se destaca como tal, se destaca respecto de la forma de su obtención. Dada en presencia - en virtud de la forma del tiempo entero- es destacarse la diferencia real. A saber: 1) Destacarse la diferencia en concentración, a resguardo de su dispersión en el tiempo. 2) Destacarse la diferencia sin eliminación de la instancia que establece su vigencia real, esto es, sin eliminación del tiempo.

Por lo tanto: en el destacarse la diferencia real, el tiempo queda concernido; pero, a la par, sin que el tiempo concernido se adose como opacidad a la diferencia -que en tal caso no se destacaría-. El tiempo de la presencia articula entero: lo entero del tiempo es la transparencia en que comparece la diferencia real. La forma inteligible es nítida, precisa, porque traspasa el tiempo.

La diferencia presente traspasa: no desparramarse es su lucir. Pero la diferencia presente es determinación real, tiene vigencia precisamente a título de ser temporal. Eso significa que este lucir o traspasar de la determinación presente no elimina el tiempo traspasado. Por lo mismo, la plenitud formal del tiempo entero remite intencionalmente a su condición antecedente. Las articulaciones parciales del tiempo -dadas en los niveles cognoscitivos inferiores- son tal condición. Sin noticia sensible del tiempo la forma que articula en presencia carecería de contenido. En suma: sin notación sensible del tiempo (contenido temporal) no cabe articulación en presencia (forma plena temporal). Sobre este comienzo se repliega el método.

Por el contrario. El método dialéctico cifra su validez en un recorrido de las direcciones metódicas concurrente en fusión o solapamiento. La primera direc-

\footnotetext{
4 Polo llama al límite mental -la articulación del tiempo entero- antecedencia. Antecedentemente dado -con referencia al objeto entendido- significa todo lo contrario que dado desde sí mismo. Antecedentemente dado quiere decir ob-tenido en virtud de la antecedencia. La antecedencia por su parte no se vuelca en obtención. La antecedencia es límite, ocultamiento: tenencia o haber -lo -que -hay, lo ob-tenido. A este mismo título la presencia mental es diferencia pura, distancia insalvable entre el contenido real obtenido y la forma metódica de su obtención. Como la forma del saber es esta misma diferencia, el método solo coincide con la diferencia real en la medida de su salvaguarda.
} 
ción metódica resulta exhaustivamente recorrida. Pero esta primera dirección no admite tal recorrido exhaustivo, puesto que su cometido es la fijación del comienzo intelectual. La fijación del comienzo reduce el tiempo a su forma inteligible plena. Fijar el comienzo significa retroceso metódico porque equivale a echar cuentas, a ajustar el comiendo dado. En este sentido, fijar el comienzo es a la par retroceso porque el ajuste arroja un saldo. Pero si el comienzo es fijo, el ajuste solo cabe como explicitación de continuidad intencional Es decir: la articulación del tiempo entero no subsume -sino que versa sobre- las articulaciones temporales parciales. La explicitación retrospectiva de semejante continuidad es el genuino cometido de la primera dirección del método.

En cambio Hegel no sólo confina el tiempo a su forma inteligible plena sino que procede a la reducción negativa de esta última. El solapamiento corre a cargo de la segunda dirección -el pensamiento negativo- que absorbe la función de la primera hasta convertir en exhaustivo su recorrido. Esa exhaustividad se traduce en invasión sustitutiva de las articulaciones parciales -sensibles- del tiempo.

El pensamiento negativo es hegemónico en Hegel, ${ }^{5}$ acapara, hasta la eliminación -y ulterior reposición- de la determinación directa. Como en seguida veremos, el tiempo queda absorbido en el comienzo metódico absoluto. De tal modo, no obstante, que esa absorción deje espacio libre a la edición ulterior (en sentido lógico, no temporal) del plexo de las configuraciones puras del tiempo. La idea absoluta se realiza de acuerdo con estas últimas.

Naturalmente, en la Ciencia de la Lógica ${ }^{6}$ ni las configuraciones temporales concernidas ni el orden de su concatenación es, propiamente hablando, temporal. No solo el comienzo sino todo el proceso lógico es atemporal: es contemplación, por así decir, silente, retrospectiva, abarcante de la totalidad del proceso. Mientras el proceso está en curso no cabe su contemplación retrospectiva; ésta última es presencia absoluta abarcante.

De otra parte, sin embargo, puede decirse - y el mismo Hegel lo dice- que de nada se habla en la Ciencia de la Lógica que escape o se ponga aparte de la realización temporal: «Lo real es desde luego distinto del tiempo, pero de manera igualmente esencial todo es idéntico al tiempo (...) únicamente lo natural está sometido, por consiguiente, al tiempo, en tanto es finito; lo verdadero por el contrario, la idea, el espíritu, es eterno.- El concepto de eternidad, sin embargo, $157 \mathrm{ss}$.

5 Cfr., Polo, L. Curso de teoría del conocimiento, III, EUNSA, Pamplona, 1988, pp.

6 Hegel, G.W.F. Wissenschaft der Logik, Gesammelte Werke, Hrg. Der RheinischWestfälischen Akademie der Wissenschaften, Felix Meiner, Hamburgo, 1968-: citaré esta obra $W L$ por la edición de Duque, F. (ed. y trad.), Ciencia de la lógica, I: La lógica objetiva, Universidad Autónoma de Madrid, Abada Editores, 2011. Para una lectura de la Ciencia de la Lógica desde la perspectiva filosófica de Polo cfr., Padial, J.J., La idea en la ciencia de la lógica de Hegel, Universidad de Málaga, 2003. 
no debe ser negativamente aprehendido tal como la abstracción del tiempo, a saber, como si la eternidad existiera de alguna manera fuera del tiempo; en cualquier caso, no en el sentido de que la eternidad venga después del tiempo; de este modo la eternidad se convertiría en futuro, o sea, en un momento del tiempo»?

El tratado lógico explicita el regreso contemplativo de la Idea sobre su propio fondo, prescindiéndose aquí de la realización temporal. Claro está: salvo por lo que se refiere al fundamento y necesidad conceptual de esta misma realización. En este preciso sentido hay que decir que la dirección negativa del método absorbe a la primera sin dejar de reponerla.

En cuanto a la tercera de las direcciones, la fusión con las otras reviste un carácter peculiar, en razón de que también para Hegel se trata de la dirección metódica más excelente. La libertad trascendental vale en Hegel como instancia ponente del método. La expresión introduce contraste con la anteriormente empleada: instancia exponente del método. La doble consideración de la libertad en Hegel -negativa y realizativa- responde al doble movimiento con que se solapan las direcciones del método: «La forma suprema de nada sería de suyo la libertad, pero ésta es la negatividad en tanto se sumerge en su propia profundidad hasta la máxima intensidad, y es ella misma afirmación, precisamente absoluta». ${ }^{8}$

El método hegeliano conforma un círculo, cuyo recorrido admite que se siga uno u otro de sus posibles trazados. El pensamiento negativo absorbe la dirección metódica que articula el tiempo: reduce la forma del devenir intuido a forma inteligible del estar uno después de otro, del uno repelente en exterioridad. De este modo da cuenta Hegel de la intuición pura kantiana: el elemento formal puro de la representación queda, en cuanto pensado, reducido.

El tránsito hacia la libertad ponente puede enfocar con más o menos detalle el componente subjetivo del método. En la Fenomenología del Espíritu ${ }^{9}$ la reducción negativa del tiempo cosmológico queda dialécticamente transferida al yo que conjuga el tiempo negativo del espíritu. El yo como tiempo es la negatividad que culmina en libertad absoluta, contemplación retrospectiva del proceso. En la Ciencia de la Lógica - como en la exposición preliminar de la Enciclopedia- no se aborda el acontecer temporal de facto porque el interés se centra en la forma pura del método. ${ }^{10}$

7 Hegel, G.W.F., Enzyklopäedie der philosophischen Wissenschaften im Grundisse, Heidelberg, 1817 ( $2^{\mathrm{a}}$ edición, Berlín 1827; 3 a edición, ibíd. 1830. Citaré la versión castellana de VALLS, R. (ed. y trad.), Enciclopedia de las ciencias filosóficas en compendio (en adelante $E N C)$, Alianza Editorial, Madrid, 1997, § 258, p. 317.

8 ENC, \& 88, p. 190.

9 Cfr., Haya, F., «Los sentidos del tiempo en Hegel», Studia Poliana, 9 (2007), 67-102.

$10 E N C, \S 25$, p.132. 


\section{LA FORMA METÓDICA DEL COMIENZO}

Introduce el primer libro de la Ciencia de la Lógica ${ }^{11}$ un tratamiento esquemático del problema del comienzo. La exposición detallada de este esquema es ofrecida por Hegel en la parte preliminar de la Enciclopedia de las ciencias filosóficas. Estas últimas páginas contienen una presentación escolar relativamente asequible de la doctrina hegeliana del método de la filosofía.

La heurística puramente constructiva de Hegel prohíbe el tanteo. De ahí que Hegel asiente en bloque el lugar de la Lógica dentro del sistema. La Lógica se introduce, por decirlo así, toda entera, en la silenciosa región del pensamiento, ${ }^{12}$ el lugar donde carece de dominio el tiempo.

El comienzo absoluto es la posición absoluta del método. En contraste con la secuencia del tiempo, el despliegue metódico forma un círculo. Considerado en función de la forma del saber, el tiempo se anula en el concepto. ${ }^{13}$ Cae también así la incompatibilidad entre progreso lineal y circularidad metódica. El fundamento lógico se inserta en cualquier tramo, en cualquier punto de la realización temporal, por la misma razón que sostiene a la serie completa. Por eso el orden metódico incluye a lo primero tanto en el sentido del principio como en el de comienzo: «el reflexionar en general contiene en primer lugar el principio de la filosofía (también en el sentido de comienzo)». ${ }^{14}$

La distinción entre el principio de la filosofía y su comienzo evita que lo primero en la secuencia temporal usurpe la prioridad jerárquica en sentido propio. Lo primero en sentido propio -absoluto- es el principio, del que se ocupa el método. El método es la forma misma del saber temáticamente considerada, esto es, en condición exenta o refleja. Puede añadirse que para Hegel, la forma del saber es la forma en sentido propio, la forma como tal. La forma como tal solo llega a serlo en reversión, en reduplicación.

El comienzo absoluto concierne a la posición del método. Aquí se prescinde directamente -a la par que indirectamente se incluye -el comienzo temporal. El comienzo absoluto mienta en directo el principio, y solo derivadamente el comienzo, en cuanto englobado o incluido. A semejante comienzo absoluto equivalente al principio- alude el reproche de Hegel a sus predecesores modernos: «sobre todo en los tiempos modernos, el tener que encontrar un inicio en filosofía vino a verse como una dificultad, y se ha hablado mucho y variado sobre la razón de esta dificultad, así como de la posibilidad de resolverla. El inicio de la filosofia tiene que ser, o algo mediado, o algo inmediato; y fácil es

11 Cfr., $W L$, pp. 215-222.

$12 \mathrm{La}$ expresión es de Hegel, en el prefacio a la segunda edición de $W L$.

13 Cfr., Heidegger, M., Hegels Phänomenologie des Geistes, GA 32, Klostermann, V., Frankfurt, 1980. Trad. esp. de M. Vázquez y K. Wrehde, La fenomenología del espiritu de Hegel, Alianza Editorial, Madrid, 1995, p. 26.

14 ENC., §7, p. 107. El paréntesis es de Hegel. 
mostrar que no podría ser ni lo uno ni lo otro, con lo que una y otra manera de establecer el inicio encuentran su refutación». ${ }^{15}$

Según Hegel la posición parcial, descoyuntada, del comienzo es aporética. El método se detiene en la aporía del comienzo: «Si la filosofía ha de ser ciencia no puede tomar prestado al efecto su método, según he recordado ya en otro lugar, de una ciencia subordinada, como es la matemática, ni tampoco darse por satisfecha con aseveraciones categóricas de la intuición interna o servirse de una argumentación raciocinante fundada en la reflexión externa. Aquí sólo puede tratarse de la naturaleza del contenido, la cual se mueve en el conocimiento científico, en cuanto que, al mismo tiempo, es esa reflexión propia del contenido la primera en poner y engendrar la determinación misma de éste». ${ }^{16}$

El comienzo sistemático de la filosofía es la posición de su método: el saber adquiere aquí su forma verdadera, el principio descansa por fin en su propio elemento. Pero resulta que «no sólo se ha discutido mucho sobre la dificultad (del problema del comienzo) sino que se ha planteado la posibilidad misma de su solución». ${ }^{17} \mathrm{La}$ imposibilidad de solución del problema del comienzo es la aporía que detiene al método. El avance se despeja pues como esclarecimiento del proceder metódico: tal es el significado de posición absoluta del método, del venir a sí misma de la forma del saber.

Eso es también la completa exención de supuestos. El contenido del comienzo absoluto es la comparecencia exenta - en exclusiva- de la forma pura del método. Lo excluido es la referencia de esta forma a contenido alguno ajeno, que mancillara su trasparencia. Cualquier contenido determinado ensuciaría la fuente de la determinación inteligible: «La idea absoluta, porque en ella no hay ningún pasar ni ningún presuponer, ni hay en general ninguna determinidad que no sea fluida y transparente, es para sí la forma pura del concepto que intuye $s u$ contenido como ella misma. Es para sí contenido en tanto ella es el distinguir ideal de sí misma respecto de sí y uno de los distintos es la identidad consigo, en la cual sin embargo está contenida la totalidad de la forma como el sistema de las determinaciones de contenido. Este contenido es el sistema de lo lógico. Como forma no le queda aquí a la idea nada más que el método de este contenido: el saber preciso del valor garantizado de sus momentos»». ${ }^{18}$

La posición absoluta del método es la reflexión con que se origina lo sabido. Por lo mismo, es el despojo de lo sabido hasta el elemento en que saber es mero impulso. De otro modo la forma del saber se empaña, en lugar de avanzar desliza hacia el afianzamiento hurtado: «Solamente en y por esta pura inmediatez es el ser nada, un inefable; su distinción respecto de la nada es mera opinión. Pro-

15 WL, pp. 215-216.

16 WL, pp. 185.

17 WL, pp. 215-216.

$18 E N C, \S 237$, p. 296. 
piamente se trata solamente de la conciencia de esos comienzos, es decir, que ambos no son más que abstracciones vacías y uno es tan vacío como el otro; el impulso a encontrar en el ser o en ambos un significado firme es la misma necesidad que impulsa hacia adelante al ser y a la nada y les confiere un significado verdadero, es decir, concreto. Este ir hacia adelante es la realización lógica y el transcurso que se presentará en lo que sigue. La reflexión que encuentra para estos comienzos determinaciones más profundas es el pensamiento lógico, mediante el cual tales determinaciones se producen, no de manera contingente, sino necesaria». ${ }^{19}$

La aporía de principio es ante todo, según Hegel, la aporía del comienzo. Llamo aporía de principio a la tematización del método que revierte como su colapso, como detención de su impulso. Hegel alude expresamente a la detención que malogra al método vacilante en su comienzo: «no sólo se ha discutido mucho sobre la dificultad sino que se ha planteado la posibilidad misma de su solución». ${ }^{20}$

La aporía del comienzo es la apariencia según la cual el problema del principio del saber es insoluble. El comienzo de la filosofía es ciertamente problemático. Hegel no niega este extremo; al contrario, lo subraya hasta el colmo. Pero lo problemático del saber no equivale a su aporía. La aporía es el enunciado problemático bajo la forma metódica que cierra el paso. Es la proyección del fracaso metódico como insolubilidad del problema temático.

La aporía no está en el contenido problemático sino en la forma defectuosa de su planteamiento: «Como sea que el espíritu, en cuanto siente e intuye, tiene a lo sensible por objeto, en cuanto es fantasía tiene imágenes, en cuanto voluntad fines, etc., también procura él satisfacción a su suprema intimidad, al pensamiento, oponiéndolo a esas formas de su existencia o simplemente distinguiéndolo de ellas, y gana al pensar como objeto suyo. De este modo se encuentra a sí mismo en el sentido más profundo de la expresión puesto que su principio, su mismidad sin mezcla, es el pensar. En este negocio suyo ocurre, sin embargo, que el pensar viene a dar en contradicciones, es decir, se pierde en la noidentidad solidificada de los pensamientos, con lo cual no se alcanza a sí mismo, sino que más bien permanece cogido por su opuesto». ${ }^{21}$

Tal estar cogida de la forma del pensamiento es la aporía de su contradicción irresuelta. Por eso es aquí, en el lugar al que el pensamiento se retira para cobrar su impulso, dónde a la par encuentra, con la verdadera forma de su intimidad, que es también su más profundo problema, su desgarramiento. El problema no es ilusorio, entiende Hegel, porque en verdad se desgarra el pensar en su necesa-

19 ENC, § 87, p. 190.

20 WL, pp. 215-216.

$21 E N C, \S 11$, p. 113.

Suplemento 19 (2014) de Contrastes. Revista internacional de filosofía 
rio adelantamiento. Comenzar a pensar es, anticipación en precario; de ahí que el problema se agudice en la dirección en que pensar retrocede hacia su centro.

Pero la aporía del comienzo no es la forma definitiva del problema del saber justo si la precariedad es adelantamiento. Por lo tanto, según Hegel, en el lugar del falso afianzamiento en precaria anticipación, el método pone el procedimiento inverso: insiste en el despojo de la determinación que se anticipa hasta nivelar su precariedad. Nivelar la precariedad de la determinación directa es despojarla, es decir, conducirla al nivel de su verdadera indigencia. Entonces también el saber deja de ser problema puesto que éste se hacía consistir en la exigencia del adelantarse, equivalente al concernir del saber a lo ya determinadamente sabido (distinto del saber mismo). La indigencia del despojo metódico es pura puro saber de nada, mero impulso- pero precisamente por ello, no equivale ya a precariedad, sino al contrario: afianzamiento del comienzo en la forma pura del método.

La apariencia aporética del problema del comienzo se desvanece como figura de un tránsito necesario: «La insatisfacción más alta se dirige contra este resultado del pensar meramente intelectivo y, procediendo así, se apoya en que el pensar no se suelta de sí y permanece fiel a sí mismo incluso en esta pérdida consciente de su ser-cabe-sí «hasta que vence» y lleva a cabo en el pensar mismo la resolución de sus propias contradicciones».. ${ }^{22}$

El enunciado de la aporía del comienzo es la escisión disyuntiva del dilema siguiente: «O se comienza por lo inmediato $o$ se comienza por lo mediato». ${ }^{23}$ Ninguno de los disyuntos - dice Hegel-, hace justicia a la verdadera forma del método, que es también su principio: «Fácil es mostrar que (el comienzo) no podría ser ni lo uno ni lo otro, con lo que una y otra manera de establecer el inicio encuentran su refutación». ${ }^{24}$

En la consideración de Hegel, la forma inadecuada del método puede llamarse lineal, mientras que la adecuada, circular. Solo una forma metódica circular propicia el comienzo absoluto. La falsa inmediatez del comienzo es fácilmente refutable -según Hegel- en razón de su lineal incompatibilidad con la mediación del proceso. En cambio, la circularidad metódica suprime el aparente conflicto entre disyuntos. No hay tal dilema -o se comienza por lo inmediato o se comienza por lo mediato- porque «si bien ambos momentos aparecen también como distintos, ninguno de los dos puede faltar y ambos están bajo un vínculo inseparable». ${ }^{25}$

El vínculo al que Hegel se refiere es la forma circular del método, de acuerdo con la que también se establece el comienzo absoluto: «Por lo que se refiere al

22 Ibid.

23 WL, pp. 215-216.

$24 \mathrm{Ibid}$.

$25 E N C, \S 12$, p. 114.

Suplemento 19 (2014) de Contrastes. Revista internacional de filosofía 
comienzo que la filosofía tiene que adoptar, parece que ella comienza con una suposición subjetiva, igual que las otras ciencias en general, a saber, con un objeto particular, y así como en los otros casos se toma al espacio, al número, etc., parece que aquí hay que tomar al pensamiento como objeto del pensar. Sólo que eso es precisamente el acto libre del pensar: ponerse en la posición en la que es para sí y, por tanto, él mismo se engendra y da su objeto. Más adelante, aquella posición que de este modo aparece como inmediata, debe convertirse en resultado en el interior de la ciencia y precisamente en su último resultado con el cual la filosofía alcanza de nuevo su comienzo y a él regresa». ${ }^{26}$

Y, no obstante, según Hegel, el comienzo metódico es el absoluto inmediato. El dilema sobre la posición -inmediata o mediada- del comienzo deriva de que el comienzo declarado inmediato no es tal. De ahí la paradoja, expresa en el último párrafo de la Introducción al primer libro de la Lógica. El comienzo absoluto es el ser indeterminado, la pura inmediación, no contenido mediado alguno, de manera que «este inicio -el comienzo absoluto- no precisa de preparación alguna ni introducción ulterior; y este preámbulo raciocinante sobre él no podía tener intención de hacerlo presente, sino más bien de alejar todo preámbulo». ${ }^{27}$

Hegel no pretende pues demostrar el comienzo absoluto de la ciencia. El comienzo se llama absoluto precisamente porque está desligado de cualquier referencia externa o extraña. El comienzo como tal no precisa de preparación ni admite demostración. La indemostrabilidad del comienzo equivale a su absoluta inmediatez.

Pero entonces: ¿por qué ha argüido Hegel -en esta misma Introducción- contra el dilema que comprende, como uno de sus disyuntos, el enunciado del comienzo inmediato? La respuesta es obvia: la inmediatez que se refuta no es la genuina. Precisamente por ello esa supuesta inmediatez se muestra incompatible con la mediación del proceso lógico. La fórmula misma del dilema - el comienzo es inmediato $o$ mediato- es claro índice de que no se ha obtenido la verdadera inmediatez del comienzo. La fórmula del dilema es el enunciado lógico de la aporía del comienzo, es decir, de su problema irresuelto. Pues en su verdadera inmediatez, el comienzo absoluto contiene el movimiento de su propia mediación.

El comienzo absoluto es indemostrable porque su inmediatez es pura irreferencia: ausencia de exterioridad, de remitencia a lo antecedente o ajeno a la propia interioridad. La obtención del comienzo absoluto invierte el sentido direccional ordinario que se concede a la demostración. Demostrar no es el afianzar mediado en lo evidente -externo y anterior- sino el entero volcarse hacia delante: justificación según el implementarse del impulso. El comienzo es indemostrable a título de comienzo absoluto de la demostración.

$26 E N C, \S 11$, p. 113.

27 WL, p. 222.

Suplemento 19 (2014) de Contrastes. Revista internacional de filosofía 
Como tal comienzo no escinde su absoluta inmediatez con relación al proceso lógico: no se adelanta parcialmente ni es su alternativa, sino la misma forma vacía del proceso. La aporía del comienzo se tendía sobre la rigidez con la que a la inmediatez se opone la mediación. Esta rigidez-característica de la representación- equivale a fracaso del pensamiento en la elevación de la inmediatez hasta su concepto. En el puro despojo del ser indeterminado comparece en cambio la forma del método. Comenzar es lanzar el pensamiento desde la vaciedad de su contenido determinado.

Sopesemos brevemente la distancia entre la posición de Hegel y la de Aristóteles en la respectiva defensa indirecta o argumentativa del principio metódico. El principio aristotélico de no contradicción sienta la antecedencia no problemática del comienzo. No solo rechaza la aporía del comienzo, sino con ella excluye la consideración problemática del conocer, considerada en sentido positivo. Hegel en cambio da plena carta de naturaleza a esta problemática, porque el método negativo es la solución positiva al problema -también positivo- del escindirse de la intelección respecto de sí: «la negación de la negación no es una neutralización; lo infinito es lo afirmativo y solamente lo finito es lo superado». ${ }^{28}$

En cambio, dice Aristóteles: es imposible postergar lo anterior. ${ }^{29}$ Lo anterior es exterior al proceso en el sentido de lo evidente que antecede, lo no susceptible de enunciado problemático. Para Hegel no hay tal anterioridad que escape al proceso. A la inversa que Aristóteles, Hegel dirige su argumentación refutativa a eliminar cualquier fijeza de contenido ya dado o antecedente. Considera Hegel que solo así el proceso despeja su avance.

El segundo disyunto del dilema que Hegel rechaza enuncia: el comienzo es inmediato. Naturalmente, debe leerse: comienzo inmediato en mostrenca oposición al proceso lógico. En relación con este disyunto, la argumentación de Hegel quiere impedir la intromisión en el inicio de cualquier contenido determinado. Cualquier diferencia añadida a la indeterminación vacía del ser invalida el comienzo como tal. La forma del comenzar es tanto ser como nada, puro impulso, simple volcarse sin supuesto.

El comienzo no puede contener determinación alguna. Hegel excluye expresamente la interpretación que pusiera el comienzo en la Fenomenología del Espiritu. ${ }^{30}$ Este último sólo en apariencia es inmediato, porque en verdad la inmediatez sensible resulta doblemente mediada, tanto del lado de la cosa como del lado de la conciencia. La intuición sensible del tiempo a que se refiere Kant, expone de modo simple, a juicio de Hegel, aquella faceta preliminar de la cuestión cuya verdad ha de ganar la reflexión.

28 ENC, § 95, p. 199.

29 Metaph .III, 1, 995a 33-34.

30 Cfr., $W L$, p. 216. 


\section{LA REDUCCIÓN NEGATIVA DE LA FORMA TEMPORAL}

Examinemos el juego de la articulación del tiempo en orden al comienzo absoluto del saber. La posición de Hegel se perfila en contraste con la de Kant. De entrada: el tiempo de la secuencia es el orden formal de la experiencia. Hegel no niega este extremo; es decir, concede a Kant su porción de acierto: todo conocimiento comienza con la experiencia. Para la conciencia subjetiva el saber es adquirido en secuencia temporal. En el marco del desenvolvimiento de la experiencia llega a hacerse consciente la idea absoluta. Pero el dominio íntimo de esta última es silenciosa región que deja fuera el tiempo. El tiempo de la secuencia no asiste al comienzo absoluto del método.

Examinaremos el relativamente breve pero denso compendio de la Enciclopedia. Dice Hegel: «La negatividad, que como punto se refiere al espacio y en él desarrolla las determinidades de ella como línea y superficie, en la esfera del ser-afuera-de-sí es también ciertamente para sí juntamente con sus determinaciones, pero a la vez, siendo ponente en esta esfera del ser-afuera-de-sí, está apareciendo como indiferente ante el quieto uno-junto a-otro. Puesta de este modo para sí, la negatividad es el tiempon. ${ }^{31}$

El tiempo es la negatividad, dice aquí Hegel: la negación puesta en la esfera del ser-afuera-de-sí, y a la par indiferente a la negatividad expuesta como espacio. En cuanto a esta indiferencia (no-diferencia) entre los respectivos valores negativos de la exterioridad en tiempo y espacio, leemos un poco más arriba: «Sobre la naturaleza del espacio se han dicho muchísimas cosas desde siempre. Recordemos solamente la determinación kantiana según la cual el espacio, como el tiempo, es una forma de la intuición sensible. También en otros autores se ha impuesto la costumbre de dar por sentado que el espacio sólo debe ser contemplado como algo subjetivo en la representación. Si se prescinde de lo que en el concepto kantiano corresponde al idealismo subjetivo y a sus determinaciones, queda como resto la determinación correcta de que el espacio es una mera forma, esto es, una abstracción, y precisamente la abstracción de la exterioridad inmediata». ${ }^{32}$

La indiferencia entre las respectivas determinaciones negativas del tiempo y del espacio está en su común valor de forma abstracta, según el significado que Hegel suele otorgar a este último adjetivo: forma abstracta es forma vacía: «El tiempo es tan continuo como el espacio, puesto que es la negatividad refiriéndose a sí abstractamente y bajo esta abstracción no se da aún ninguna distinción real»». ${ }^{33}$ Tanto espacio como tiempo son formas que prescinden del contenido, de la diferencia de realidad que albergan. El acierto de Kant es depurado de su lastre idealista subjetivo si se considera que espacio y tiempo, además de formas

$31 E N C, \S 257$, pp. 315-316.

$32 E N C, \S 254$, p. 313.

$33 E N C, \S 258$, p. 316 . 
de la intuición son formas categoriales objetivas. Su significado conceptual es la exterioridad abstracta del ser. Según esa exterioridad el ser es intuido -es inmediato-, o más bien, de acuerdo con esa exterioridad el ser mismo es intuición.

Hegel propone pues esta definición del tiempo: «El tiempo, en cuanto unidad negativa del ser-afuera-de-sí, es igualmente algo simplemente abstracto e ideal. Es el ser que siendo, no es, y que no siendo, es; es el devenir intuido, es decir, que las diferencias precisa y simplemente momentáneas o que inmediatamente se superan, están determinadas como exteriores, esto es, como exteriores a $s i$ mismas, sin embargo»». ${ }^{34}$

La definición es compleja. Admite el desglose siguiente:

\section{III.1. EL TIEMPO ES LA UNIDAD NEGATIVA DEL SER-AFUERA-DE-SÍ}

El tiempo es la negación pensada como el anudarse de la exterioridad del ser. La diferencia de esta forma unitiva es negativa porque su principio es el uno que se repele de sí mismo y vuelve como nudo de esa misma pluralidad de unos. El uno principio de la serie y a la par de su articulación formal -el tiempo-, es, según hemos leído, la negatividad ponente en la esfera del ser-afuera de sí.

El uno negativo es para sí junto con sus determinaciones: este para sí alude al desarrollo precedente - a la mediación- respecto del uno. El uno negativo no es de suyo inmediato, si bien -resalta el primer texto- aparece como tal en su asimilación al espacio, esto es como lo inmediato exterior abstracto. La inmediatez abstracta común a espacio y tiempo se cifra en la común carencia de contenido inteligible aparte de esta pura forma de la exterioridad.

\section{III.2. EL TIEMPO ES EL SER QUE SIENDO, NO ES, Y QUE NO SIENDO, ES}

Esta conocida descripción puede tomarse en un sentido más general, y en otro más preciso que entra a considerar las categorías en la expresión implicadas.

En sentido amplio, -concerniente al tiempo en bloque - significa que en tiempo se resuelve la realidad toda del acontecer; de este modo del tiempo mismo puede decirse: a) Que es el ser que siendo no es, puesto que todo acontecer es realidad declinante, pasar. b) Que es el ser que no siendo es, porque en el tiempo - carente de contenido real determinado - se resuelve toda la realidad del acontecer.

De acuerdo con esta última lectura confrontemos la cita que ya incluí antes: «Lo real es desde luego distinto del tiempo, pero de manera igualmente esencial todo es idéntico al tiempo». ${ }^{35} \mathrm{Y}$, un poco más arriba: «En el tiempo, se dice, surge y perece todo; y cuando se abstrae de todo, es decir, de lo que llena el

34 Ibid.

35 ENC, $\S 258$, p. 317.

Suplemento 19 (2014) de Contrastes. Revista internacional de filosofía 
tiempo, como cuando se abstrae de lo que llena el espacio, resta el tiempo vacío igual que el espacio vacío. O sea, que entonces estas abstracciones de la exterioridad están puestas como si fueran de suyo algo. Pero no es en el tiempo donde surge y perece todo, sino que el tiempo mismo es ese devenir, ese surgir y perecer, el abstraer que-está-siendo; es Cronos que lo pare todo y devora sus partos».. 36

Resulta interesante notar -en este último texto- la manera en que Hegel evade la suposición del tiempo. Abstraído todo contenido real, queda el tiempo vacío: pero no es el tiempo el marco en que todo acontece, sino que el tiempo es ese mismo acontecer, lo real como acontecer. A la par se señala en el texto la identidad entre la forma de la representación y la forma de la exterioridad inmediata del ser: el abstraer que-está-siendo.

Pero el tiempo así descrito, como el ser que siendo, no es, y que no siendo es, exige un análisis más preciso que explicite las categorías concernidas. Ser que pasa a no ser y no ser que pasa a ser es devenir. La forma del pensamiento que el tiempo explaya es el devenir, pero de tal suerte que esa configuración queda también acotada como lo exterior del ser-fuera-de-sí en inmediatez. De ahí la coincidencia indicada entre la unidad negativa del uno en repulsión y la forma del devenir intuido.

\section{III.3. EL TIEMPO ES LA FORMA DEL DEVENIR INTUIDO}

La intuición significa la forma de la inmediatez sensible con que se cancela la mediación que recorre al tiempo. Pero el tiempo, según su concepto, no es inmediato, sino la negación ponente y contraída del uno en repulsión. Hegel afirma expresamente: «La idea que es para sí, considerada según esta su unidad consigo, es intuir; y la idea que intuye, naturaleza. En cuanto intuir empero, la idea está puesta bajo la determinación unilateral de la inmediatez o de la negación mediante reflexión extrínseca». ${ }^{37}$

Insisto en que la reducción negativa de la forma temporal equivale en Hegel a su deducción negativa. Por eso lo inmediato de la forma temporal -que es también lo inmediato de la experiencia- no equivale sin más a la inmediatez del comienzo absoluto. De otra parte, la inmediatez sensible vertida a la unidad negativa del uno repelente -es decir, trasvasada a concepto- no obtiene otra determinación inteligible que la exterioridad negativa del ser. De ahí que el pensamiento negativo resbale -por decirlo así- sobre esta exterioridad. Este resbalar es la determinación unilateral de la reflexión negativa extrínseca, y también según Hegel- el estatuto del pensamiento matemático sobre la naturaleza.

36 Ibid

37 ENC, § 244, p. 299.

Suplemento 19 (2014) de Contrastes. Revista internacional de filosofía 
La infinitud del tiempo se cifra en su indefinido y homogéneo extenderse. Pero Hegel insiste mucho en que ésta no es la verdadera infinitud. ${ }^{38}$ La verdadera infinitud requiere el tránsito dialéctico desde aquella exterioridad indefinida hacia la determinación del contenido pensado. Por eso también, y a este respecto, el tiempo infinito es indiferente con relación al espacio mostrenco. Uno y otro no significan sino la pura exterioridad del ser, puesta como forma del devenir e idéntica con la unidad negativa de la representación sensible.

La deducción lógica de la forma temporal en Hegel es sumamente alambicada. Las categorías que sustentan la noción del tiempo se enroscan en círculos cada vez más estrechos. En la Ciencia de la Lógica se discuten las antinomias kantianas de la cosmología dentro del lugar reservado a las categorías de la cantidad. ${ }^{39}$ El tiempo queda asimilado al número cuyo principio es el uno con arreglo al que se establece la magnitud y con ella el cuanto.

Hegel concede pues la razón a Kant, también en lo que se refiere a la tesis de que el tiempo es el principio formal puro de la matemática. En este sentido, cabe establecer analogía entre el uno que es principio del tiempo y el uno que es principio del número. Y, no obstante, añade Hegel: «tal aptitud la recibe el principio del tiempo solamente cuando se paraliza, cuando su negatividad es depuesta por el entendimiento hasta un uno. Este uno muerto, que es la suprema exterioridad del pensamiento, es capaz de combinación exterior, y estas combinaciones a su vez, las figuras de la aritmética, son capaces de determinación por el entendimiento según igualdad y desigualdad, de identificación y distinción»». ${ }^{40}$

El tiempo mostrenco de la naturaleza es la esfera formal dentro de la que se establece el juego de la unidad cuantificada consigo misma, en los precisos términos en que también cabe dar cuenta de la expresión forma del devenir in-

38 Tal es, mi modo de ver, el motivo principal, de su encono contra lo que él considera una insufrible petulancia de parte de los físico-matemáticos. Dice a este propósito que el análisis matemático toma prestadas sus nociones -que en verdad no comprende- de la filosofía. La verdadera infinitud no es el extenderse isomorfo del tiempo de Newton, que ciertamente se asimila a la infinitud de la serie numérica, y con la que es -como ya advirtiera Kant- la misma cosa.

39 En la Enciclopedia Hegel resume así su crítica al planteamiento de la segunda antinomia kantiana: «La antinomia del espacio, del tiempo o de la materia con respecto a su divisibilidad hasta lo infinito o a su composición por indivisibles, no es más que la afirmación de la cantidad, una vez como continua y otra vez como discreta. Si el espacio, el tiempo, etc., se ponen solamente bajo la determinación de la cantidad continua, son entonces divisibles hasta lo infinito; pero bajo la determinación de la cantidad discreta quedan divididos en sí y consisten en unos indivisibles; tan unilateral es lo uno como lo otro» (ENC, § 100, p.202). En la Ciencia de la Lógica discute extensamente esta antinomia en la $2^{\mathrm{a}}$ observación al cap. I de la secc. $2^{\text {a }}$ (ed. cit., pp. 298-306). La primera antinomia de Kant es refutada, desde la perspectiva dialéctica, brevemente en la $4^{a}$ observación al cap.I de la secc. $1^{\text {a }}$ (ed. cit., pp. 237-238); y más extensamente en la observación $2^{\mathrm{a}}$ a sección II, cap. II, C, 2 (ed. cit., pp. 335-339).

$40 E N C, \S 259$, p. 318. 
tuido: «El número es pensamiento, pero es el pensamiento como un ser perfectamente exterior a sí mismo. No pertenece a la intuición porque es pensamiento, pero es el pensamiento que tiene la exterioridad de la intuición como determinación suya». ${ }^{41}$

Así se entiende también la siguiente afirmación de Hegel: «El tiempo es, como el espacio, una forma pura de la sensibilidad o del intuir; lo sensible nosensible». ${ }^{42}$ Pasamos a su glosa:

\section{III.4. EL TIEMPO ES LO SENSIBLE NO SENSIBLE}

El tiempo, de suyo, como el número, no pertenece al orden de la intuición, porque es pensamiento. Pero el concepto del tiempo, como el del número solo contiene la determinación inteligible que significa la exterioridad de la intuición. En este último sentido el tiempo es no sensible y sensible.

Ahora bien: la noción del tiempo, a diferencia de la del número, explicita la forma del devenir, que en cambio, queda detenida en la configuración matemática. En cuanto que el tiempo contiene la forma del devenir, que es el impulso negativo del método, en este sentido, el tiempo se inviste de una virtualidad metódica que ni por asomo tiene el número. En la medida en que la forma del devenir recorre todo el proceso lógico, la negatividad no queda confinada a reflexión extrínseca, sino que se convierte en fuerza impulsora del método. En suma: no cabe un único sentido del tiempo en Hegel.

El tiempo del que se habla en el contexto de las categorías de la cantidad es el que se asimila al número, el tiempo cósmico, idéntico también con la intuición pura de Kant. De otra parte, la asimilación del tiempo al número no es inútil -según la perspectiva del propio Hegel-.

En efecto, parece razonable decir que el devenir solo no baste para fijar la categoría del tiempo, aunque de otra parte, Hegel afirme que el tiempo es la forma del devenir intuido. Precisamente, este último adjetivo introduce la determinación que da paso a las categorías de la cantidad.

Esta última interpretación queda avalada por la coincidencia de Hegel con otros pensadores. Tanto Aristóteles ${ }^{43}$ como Kant asimilan el tiempo al número. Kant pone expresamente el principio de la aritmética en la intuición pura del tiempo. Aristóteles distingue entre el número numerante y la medida objetiva, y pone al tiempo en doble relación, con uno y con otra. Respecto del primero, la referencia es el alma, respecto del segundo, el movimiento circular del cielo. En todos los casos, el tiempo aparece como respectividad del movimiento con relación a sí mismo.

41 ENC § 104, p.205.

$42 E N C, \S 258$, p. 316.

43 Cfr., Phys. IV, 223 a 16-30.

Suplemento 19 (2014) de Contrastes. Revista internacional de filosofía 
Ahora bien, el devenir puro de Hegel no contiene aún esa respectividad del movimiento. Hegel dice que el devenir significa el tránsito del ser al no ser y viceversa, mientras que el tiempo - de la naturaleza- contiene exclusivamente el tránsito del uno al uno ${ }^{44}$. Por eso, este uno en su detención es también principio del número, que designa el juego del uno consigo mismo. El límite de la magnitud es el cuanto. El número establece tal relación del uno cuanto consigo mismo, asentada ya la determinación de la cualidad. Y en ello consiste la falsa infinitud. ${ }^{45}$

\section{III.5. EL TIEMPO ES, COMO EL ESPACIO, UNA FORMA PURA DE LA SENSIBILIDAD O DEL INTUIR}

Aquí concede Hegel a Kant su porción de acierto. En cuanto forma pura o $a$ priori de la intuición sensible del devenir el tiempo no se deriva de la experiencia, sino que más bien es principio formal de su conocimiento: no todo conocimiento deriva de la experiencia. Como forma de la intuición sensible, el tiempo -como el espacio- viene dado en inmediatez, o más bien, es lo inmediato mismo de la representación, el ahora con que la representación comparece. Pero, según se ha expuesto, lo inmediato no se opone a la mediación más que para el entendimiento ligado - precisamente- a la representación. De manera que la forma intuitiva de esta última es tanto condición -elemento- del contenido inmediatamente representado, cuanto susceptible ella misma de derivación, es decir, de ser pensada como resultado de mediación. Aquí introduce Hegel su línea de discrepancia respecto de Kant.

La forma de la representación sensible contrae, por así decir, en su inmediatez la mediación negativa de la que deriva y que la recorre proporcionándole su forma de unidad. La unidad del tiempo es el uno después de otro, el destacarse de lo inmediato que caduca, cuya forma de inmediatez no exhibe sino la negación reiterada del uno: uno que como tal se destaca y pasa... a uno que como tal se destaca y pasa ...etc. Luego la verdadera forma de la representación es la inmediatez con que se contrae la serie de lo negativo (objeto uno vacío) mediado (negado) respecto de sí. Uno destacado (objeto vacío) después de (no «el uno» anterior)- antes de (él mismo en decaimiento, no «él mismo» como tal uno). ${ }^{46}$

El tiempo es según Hegel, de entrada, una cierta unidad con que de manera inmediata queda para la conciencia la representación sensible. «El tiempo es, como el espacio, una forma pura de la sensibilidad o del intuir; lo sensible no-

44 Eso significa también, según Hegel, el disolverse del devenir como tal en un mero resbalar sin ganancia especulativa. Cfr. WL, pp. 277-278.

45 Cfr., ENC, § 94, p. 197.

46 Vid. la glosa de Heidegger, M., Sein und Zeit, Max Niemeyer, Tübingen, 1993 (17 edic.), \& 82; Ser y tiempo, trad. Jorge Eduardo Rivera, Editorial Trotta, Madrid, 2003, p. 442 y ss.

Suplemento 19 (2014) de Contrastes. Revista internacional de filosofía 
sensible». ${ }^{47}$ Es importante llamar la atención sobre esta doble condición del tiempo en Hegel, puesto que marca a la par el acuerdo y la discrepancia con relación a Kant. El tiempo es forma -como dice Kant-; es además forma sensible. En cuanto forma pura de la sensibilidad, el tiempo como el espacio abraza a título de condición formal cualquier contenido representado en la experiencia. Pero, de otra parte, el tiempo es también forma inteligible.

El tiempo es forma general (a saber, del devenir de lo real). En tanto unidad (de la representación), el tiempo conecta diferencias, esto es, aúna pluralidad. Pero la diferencia misma del tiempo es la de cierta pluralidad incualificada. Las diferencias conectadas son «precisa y simplemente momentáneas»; lo cual equivale a que «inmediatamente se superan». La superación inmediata de los momentos temporales alude al ejercicio no mediado o no suficientemente dialéctico de la negación. La unidad negativa lograda es abstracta o externa. El concepto recorre, por así decir, la representación sin llegar a penetrarla. La conecta exteriormente sin alcanzar a derivar de ella su contenido diferenciado.

Por lo tanto: la forma de la representación - de la intuición del devenir-, el tiempo, es tanto lo inmediato mismo de la experiencia como lo más vacío de ella, su pura exterioridad, el contraerse en el ahora de la serie que sólo contiene el tránsito -el devenir- de lo uno a lo uno, sin explicitar el contenido determinado de lo que deviene ni la forma determinada del devenir. Hegel vincula estrechamente exterioridad -secuencia del tiempo y posición mostrenca del espaciocon vaciedad, ausencia de contenido pensado (aparte del pensamiento de esta misma forma que sólo expresa el añadirse exterior). En este respecto el tiempo se asimila al número. ${ }^{48}$

El tiempo es, según Kant, ${ }^{49}$ elemental porque su análisis o división no ofrece nada distinto de la propia representación del tiempo. Eso significa que el carácter elemental del tiempo no es conceptual, puesto que no hay modo de despejar su concepto para distribuirlo entre sus particulares. Los particulares o inferiores con relación al tiempo no son el tiempo sino su división (un lapso de tiempo). De modo que el tiempo es una representación no universalizable sino en todo caso dada como una unidad particular.

La imposibilidad de la conceptualización del tiempo equivale a su inderivabilidad. La forma del tiempo es a la par sensible y pura. En cuanto tal, no susceptible de derivación. Es una representación dada a la par como condición de la representación, y en tal sentido, confusa con el contenido determinado de ésta. De ahí el carácter elemental tanto del espacio como del tiempo.

47 ENC, § 258, p. 316.

48 Cfr., ENC § 104, p.205.

49 Cfr., I. Kant, $K r V$, A 32, B 48. Este mismo argumento, con relación al espacio es detalladamente estudiado en Placencia, L., La ontología del espacio en Kant, Cuadernos de Anuario Filosófico, $\mathrm{n}^{\mathrm{o}}$ 192, Servicio de Publicaciones de la Universidad de Navarra, 2007, pp. 156ss. 
En cierto sentido, la posición de Polo sobre el tiempo es más próxima a la de Kant que a la de Hegel, siempre que se hagan importantes salvedades encaminadas a la precisión del método. En este último respecto, la posición de Hegel sobrepujaría a la kantiana. En efecto, aunque deba admitirse con Kant la condición elemental $-\mathrm{y}$ por lo tanto conceptualmente irreductible- de la noticia del tiempo, así como su indole formal entera -y por lo tanto, refractaria a la distribución predicativa del concepto general-; aunque deba concederse a Kant todo eso, hay que notar con Polo (en esta dirección más próximo a la posición de Hegel) lo siguiente: $1^{\circ}$-La forma unitiva del tiempo no puede quedar exclusivamente en el nivel intencional sensible -inferior- porque en tal caso, el método no alcanza su cota, no incoa. Recordemos que el método de la filosofía primera retrocede hasta el primer comienzo de la vida intelectual, pero el tiempo como forma sensible no alcanza a extenderse, por así decir, de manera suficiente como para propiciar el inicio metódico.

Debe admitirse en consecuencia, una unidad formal del tiempo entero, a la que Polo llama presencia o límite mental, que además resulte tácita -no confusa con el contenido de la representación-. Hegel no cesa de glosar el concepto de esta unidad entera, en la que hace consistir el saber absoluto. Si bien, como se ha expuesto, al contrario que Polo, Hegel incurre en el extremo de desplazar de la presencia el contenido real temporal: de excluir la determinación directa del comienzo metódico.

$2^{\circ}$-De otra parte, debe notarse que en Kant juega también -como más claramente en Hegel- la preeminencia del pensar negativo o modal, ${ }^{50}$ de la que precisamente es consecuente heredero el propio Hegel. Esa preeminencia se echa de ver con claridad en el mismo argumento que Kant ofrece para probar el carácter elemental, no derivable, de la intuición del tiempo. Además, tanto Kant como Hegel son herederos de la tradición racionalista, como delata el uso kantiano, en este mismo argumento, de la noción de análisis.

Descartes $^{51}$ comprende la idea clara y distinta como el elemento inteligible cuyo análisis no obtiene nota oculta alguna, en suma, cuyo análisis reitera de manera indefinida lo primero mismo que se muestra. Una idea clara y distinta es, en definitiva mono-nota, según la expresión de Polo. Parece claro que esta última noción opera en el argumento sobre la imposibilidad del análisis conceptual del tiempo -cuyos inferiores no son casos sino partes-. También opera en Hegel, aunque de otro modo: puesto que Hegel cree poder derivar la unidad del tiempo a modo de reproducción negativa de una unidad más elemental, la del uno que se repele. Hegel se sitúa pues en el mismo plano que Kant, procediendo a probar lo que este último considera imposible. No, por cierto, que el tiempo se

50 Sobre la preeminencia del pensamiento modal en Kant, vid. González A.L., «Kant: la prueba de Dios por la posibilidad» en Tópicos, 27 (2004), pp. 25-49.

51 Cfr. Polo, L. Curso de teoría del conocimiento III, ed. cit. pp. 185ss. 
distribuya predicativamente como el concepto universal (general), sino que la entera unidad del tiempo no es elemento último, puesto que el pensamiento negativo puede desarticularla y recomponerla.

Kant emplea de manera predominante el pensamiento negativo, pues -visto desde la perspectiva de Polo- no otra cosa es verter la intelección a referencia predicativa de una idea general sobre sus casos singulares. Pero tal es en definitiva el argumento kantiano sobre el carácter elemental del tiempo. Si el tiempo no admite distribución predicativa en casos, cada uno de los cuales sea un tiempo, entonces -concluye Kant- el tiempo no es un concepto. Frente a esta posición tiene razón Hegel, al menos en el sentido de admitir la articulación inteligible del tiempo.

Además, toda la crítica kantiana descansa en definitiva sobre la base del pensamiento negativo modal, como delata la solución que Kant cree encontrar para hacer compatible el carácter elemental del tiempo con la espontaneidad que piensa según el modo de la distribución predicativa. Esa solución es la deducción trascendental del yo-pienso, de la que Hegel dice -con toda razón- que no deduce nada sino que en todo caso dispone o reparte -de manera más o menos tentativa- aquello con lo que ya cuenta.

Ahora bien, una elementalidad no racional inserta a título de ingrediente trascendental del conocimiento impide la culminación del propósito idealista. Por ello Hegel discrepa de la noción kantiana de tiempo en un doble sentido: de una parte Hegel no acepta la imposibilidad del análisis del tiempo, es decir, no admite el carácter elemental del contenido dado en la intuición del tiempo. Eso significa que el tiempo mismo ha de ser derivable en función de la negación.

\section{CONCLUSIÓN: \\ EL SOLAPAMIENTO DIALÉCTICO DE LAS DIRECCIONES DEL MÉTODO}

La diferencia entre tiempo y espacio es establecida en Hegel a partir de una elevación metódica. El pensar ha de remontarse a un plano superior, con miras a discernir la diferencia del tiempo con relación a la mera forma de la intuición, atribuida también indiferentemente al espacio. Esta tesis es correlativa con la que afirma que el tiempo hegeliano tiene otro significado, aparte de la forma del devenir intuido, o bien que el significado hegeliano del tiempo sufre transformación dialéctica.

Solo desde este segundo significado cabe que la negación remonte el falso infinito que es también la indefinida serie negativa de la forma pura de la sensibilidad. En este segundo sentido el tiempo hegeliano es idéntico al yo ${ }^{52}-a$ la

52 Sobre la equivalencia hegeliana entre subjetividad y temporalidad, vid. I. FalguerasGarciá-padial, (eds.), Yo y tiempo. La antropología filosófica de G.W.F. Hegel, en Contrastes, Suplemento 15, Málaga, 2010. 
libertad negativa ponente del método-: en este punto se aproximan hasta coincidir-se funden- las direcciones del método.

Dice en efecto Hegel: «Así como al espacio no le concierne la distinción entre la objetividad y una conciencia subjetiva que se le enfrente, tampoco la tal distinción concierne al tiempo. Si estas determinaciones se aplicaran al espacio y al tiempo, el espacio sería la objetividad abstracta y el tiempo la abstracta subjetividad. El tiempo es el mismo principio que el yo $=$ yo de la autoconciencia pura, pero es el yo o el concepto puro todavía en su total exterioridad y abstracción, es decir, como el mero devenir intuido, el puro ser-dentro-de-sí como un simple venir-afuera-de-sím.. ${ }^{53}$

La distinción entre objetividad y subjetividad no concierne ni al espacio ni al tiempo. En este sentido espacio y tiempo han de excluirse del comienzo absoluto. Pero, de otra parte, dice Hegel que cabe forzar la comparación, es decir, saltar sobre las categorías que median entre uno y otro par. En tal caso el espacio se asimila a la objetividad pura, y el tiempo a la subjetividad autoidéntica. Esta subjetividad es pura unidad negativa, identidad abstracta, y por lo mismo, despojo absoluto de contenido. Intimidad absoluta del concepto, libertad negativa ponente (y en cuanto tal carente), es decir, devenir como nervio del impulso negativo; y, por fin, absoluta exterioridad del tiempo, funden en este punto sus respectivas nociones.

En el inicial habérselas del pensamiento con el problema del comienzo opera la reducción metódica del tiempo. La dimensión metódica que articula el tiempo es en efecto de principio. Esto quiere decir: sin reducción metódica del tiempo no hay comienzo filosófico. Hasta ahí puede estarse de acuerdo con Hegel. La objeción al planteamiento dialéctico se refiere más bien al modo en que Hegel pretende la reducción metódica del tiempo.

Porque de hecho, en Hegel, el tiempo es reducido, pero no sólo a su forma plena, sino que esta misma forma del tiempo entero es a su vez negativamente reducida con vistas al comienzo absoluto. Repárese en que el tiempo comprendido como sucesión lineal de momentos- prohíbe la simultaneidad entre comienzo absoluto y mediación lógica. Pero hemos visto en los epígrafes precedentes que aquella compatibilidad traduce la forma completa del método, cuya disposición es circular: «si bien ambos momentos aparecen también como distintos, ninguno de los dos puede faltar y ambos están bajo un vínculo inseparable»r. ${ }^{54}$

La dialéctica reduce el contenido del tiempo a su forma, y esta última a su articulación de parte del pensamiento negativo. El comienzo absoluto excluye la suposición que sobre sí mismo introduce el tiempo. A mi modo de ver, Hegel tiene razón en subrayar que el pensar se alza en reducción del tiempo, que el

53 ENC. § 258, p. 316.

$54 E N C, \S 12$, p. 114.

Suplemento 19 (2014) de Contrastes. Revista internacional de filosofía 
pensar solo lo es si despega su propio recorrido del indefinido deslizarse sobre sí característico del tiempo. Pero Hegel no tiene razón -a mi juicio- es su pretensión de afianzar el método en un comienzo que prescinde del contenido real temporal.

Adviértase: en efecto, es verdad que el tiempo supone el tiempo. En cuanto notativo de la forma de lo sucesivo en relación consigo, el tiempo introduce su propia suposición. En el planteamiento de Polo, la suposición del tiempo respecto de sí, su doblarse, se contrapone al valor unificante de la presencia mental. El tiempo supone el tiempo pero, al contrario: la presencia mental no se supone. La presencia no se supone porque es la misma suposición. Con tiempo hay tiempo, pero con haber no hay haber, sino precisamente lo que hay. Justo el haber es lo que no hay. El tiempo se dobla respecto de sí. El haber en cambio es la unicidad. Por lo mismo, la presencia articula el tiempo entero. Lo entero del tiempo es la unicidad. La unicidad como carácter de la presencia mental es el valor entero del tiempo que la presencia articula. Y, correlativamente: la unicidad consigna la diferencia entre presencia articulante y tiempo articulado, justo porque, aparte de su articulación entera, el tiempo se dobla.

El tiempo supone el tiempo. La articulación entera del tiempo no supone, porque es la misma suposición. Pero eso mismo quiere decir que la articulación del tiempo entero no juega vacía, se introduce en el tiempo. ${ }^{55}$

La vaciedad hegeliana de la presencia en el comienzo introduce en ella el doblarse. El tiempo es negativamente reducido a costa de doblar la unicidad. El comienzo absoluto es doble en uno y por eso no sufre el tiempo, sustituye su articulación entera. Esta última, lejos de doblez es unicidad que exhibe, quiere decirse, alberga, versa intencionalmente sobre contenido determinado, determinación directa. El tiempo supone, la suposición no. No cabe suponer la suposición: por eso la suposición es antecedencia: exhibición - en directo- de contenido temporal. La presencia se introduce en el tiempo como la conformación entera que exhibe en cuanto exime.

El comienzo absoluto reduce la articulación del tiempo entero. En Polo la plenitud del comienzo, con relación al tiempo, es suficiente pero no absoluta. La suficiencia de la articulación del tiempo entero estriba en su mismo ocultamiento, en virtud del cual se basta para exhibir. La limitación, el límite, es este mismo ocultarse, considerado como eximente del fundamento. El quedar tácito oculta el eximir que exhibe la determinación real. Por eso, tampoco cabe que lo exhibido sea entero.

La determinación primera, el abstracto, es por fuerza no pleno, no todo lo pensable. Todo es noción impertinente con referencia al abstracto. La introducción de todo en el objeto de la abstracción -en suma, el proceder de Hegel- pro-

55 Polo, L., El acceso al ser, op. cit., p.54.

Suplemento 19 (2014) de Contrastes. Revista internacional de filosofía 
voca la colisión de las dos primeras direcciones de principio. La segunda dirección del método es el ejercicio negativo modal del pensamiento.

La modalidad ejecutiva del pensar -la forma del pensamiento- cuya diferencia misma se vierte temáticamente a todo, es la negación. La colisión dialéctica entre las direcciones de principio provoca la más formidable aporética, precisamente a la medida de su ambición; también de su indudable aproximación al núcleo en que se dirime la suerte de la filosofía primera. Para la determinación primera, el abstracto, resulta impertinente la intromisión de todo. Pero el pensamiento que niega no alcanza a recubrir con su forma el contenido directamente entendido -el abstracto- . El abstracto -el concepto, en la terminología clásicano soporta ser pensado como todo. Si se fuerza la equivalencia, la aporía precipita sobre el método en la fuente de su comienzo.

Todo, en suma, no es abstracto- determinación primera-, pero tampoco es concepto exento en que confluya la dirección del pensamiento negativo. Eso significa: todo es correlato objetivo del pensar que niega el abstracto en relación con el abstracto: todo lo demás con relación a la determinación primera negada. Por lo tanto: todo lo demás no es todo; ni «todo lo demás que el abstracto» regresa- en la segunda negación- sobre el abstracto mismo -como determinación ulterior según identidad- .

Todo no es propiamente hablando abstracto ni idea general. No es objeto de la operación que niega. La idea general es el objeto de la operación negativa. Con relación al abstracto, la idea general es determinación segunda: su indeterminación es relativa, se toma con referencia a la determinación primera. Todo no se piensa pues ni en directo, ni en virtud de la negación reflexiva. ¿Cómo se piensa? Según la atención metódica con que se ajusta la diferencia entre las misma direcciones del pensar que entran en juego.

La forma diferencial del acto del pensar negativo se tematiza -a mi modo de ver- en la serie de las nociones uno, objeto puro, todo, así como en la serie de las nociones modales que reduplican las anteriores en función del pensamiento negativo. El predominio metódico de las mencionadas tematizaciones colapsa el método puesto que funde la diferencia entre sus direcciones de principio.

FERNANDO HAYA es profesor de filosofía en el IES Hermanos Machado,

Lineas de investigación:

El método de la filosofía primera, Filosofía de la temporalidad, Filosofía de Leonardo Polo, Filosofía del idealismo alemán.

Publicaciones recientes:

2014: «El estatuto del juicio como función de atenencia», en Studia Poliana, 16, 61-82.

2013: «La tensión afirmativa de las direcciones del método», en Miscelánea Poliana, 43.

Dirección electrónica: fhayasegovia@gmail.com 